\title{
Cancer immunotherapy
}

\section{Paradigms, practice and promise}

Alex Kudrin

Medical Assessor; Medicines and Healthcare Products Regulatory Agency; London, UK
Keywords: cancer vaccines, paradigm, immunotherapy, Curiel

Submitted: $02 / 22 / 13$

Accepted: 03/07/13

http://dx.doi.org/10.4161/hv.24222

Correspondence to: Alex Kudrin;

Email: koudrine@hotmail.com

Review of: Curiel TJ, ed. Cancer Immunotherapy: Paradigms, Practice and Promise. 2013. Springer New York Heidelberg Dordtrecht London. ISBN: 978-1-4614-4732-0

\section{Dr Alex Kurdrin reviews Cancer and Promise}

The successful manipulation of a cancerdirected immune response has long been a battle ground for cancer immunologists, oncologists and patients themselves. The field of cancer immunotherapies has been littered with failures and there are estimates that more than 10,000 cancer patients were treated with these products in numerous unsuccessful clinical trials. However a glimmer of hope has arisen following FDA approval of sipuleucel-T (Provenge) for the treatment of castrateresistant prostate cancer and ipilimumab (Yervoy) approval by FDA and EMA for patients with advanced melanoma. While a plethora of publications, books and electronic media publications dedicated to cancer immunotherapies have emerged in past 20 years, book edited by Tyler Curiel is of remarkable value and contribution in this field.

First of all, the authors well-outlined the history behind the endless enthusiasm and tremendous efforts made in cancer immunotherapies and defined some of the concepts, approaches and technologies that have been tried several decades ago. Occasionally, these older methodologies provided with some modest successes but despite of failures, developers have forged further enthusiasm hoping that other ways of immunostimulation or immunomodulation might become more productive. The book has summarized the principle around "old" and "new" concepts of immunotherapy and included chapters reviewing traditional (passive and active strategies to boost antitumor immunity) and then gradually moving into area of novel contemporary immunotherapy technologies. Contributors to the book had written well-structured and referenced chapters on different tumor constituents and key elements of anti-tumor immunity, such as immune signaling of $\mathrm{T}$ cells, regulatory $\mathrm{T}$ cells, myeloid-derived suppressor cells, stromal and cancer stem cells. By capitalizing on the knowledge around older and traditional yet unsuccessful cancer immunotherapy approaches, authors underscored a fundamental notion that "not enough of a good thing" is not the fundamental problem in tumor immunology and by trying to stimulate immune system using empirical approach of adding lacking cytokines, or delivering tumorspecific antigens may not succeed alone. Additional factors attributed to characteristics of three "E's": Elimination of tumor cells, Equilibrium in the immune surveillance and continuous tumor Evasion or escape from immune response and resulting in immune dysfunction constitute what authors named "too much of a bad thing." Such a fundamental shift in paradigm implies that unless cancer immunotherapy will be able to eradicate or correct dysfunctional elements of immune system, it will be impossible to achieve a full breadth of desirable outcomes with traditional approaches.

Such a profound shift in paradigm thinking implies that sipuleucel-T and, to some extent ipilimumab represent the only ultimate regulatory successes of the "older" paradigm and that further advances in immunotherapy using new paradigm approach could potentially deliver even 
better outcomes in cancer treatment. The authors went on reviewing how combination therapies and sequential approach of using traditional chemotherapies capable of eradicating the dysfunctional antitumor immune components and antitumor monoclonal antibodies along with immunotherapies could enhance clinical outcomes and increase the probability of success with novel immunotherapeutic modalities. While some other past publications reviewed the notion that combination immune-based approaches may be more potent than any strategy used in isolation, the difference with Curiel's paradigm is that it is not based on random and empirical attempts of screening different combinations but rather applying the approach of identifying combinations that are able to suppress the dysnfuctional element of immune response.
Finally, authors laid out a chapter on immune response monitoring in cancer immunotherapy studies. Published body of evidence on $\mathrm{T}$ cell regulatory and other tumor-associated immune cells suggested that quantitative measurements of cells are not sufficient and predictive of clinical responses. Much more comprehensive and systematic characterization of immune parameters using both qualitative and semi-qualititative approaches should be developed and integrated into clinical trials.

Overall, this well-structured book is a summary of contemporary thinking about cancer immunotherapy, with a focus on the relationship between tumors and the immune response of the host. It reflects the nascent synthesis of different immunotherapy-related disciplines. The chapters are written by highly respected key thought leaders, and the topics span much of the breadth and depth of this evolving field.

The book would have benefited from a thorough discussion of regulatory and clinical development challenges with cancer immunotherapy products and what could be done from policy makers' perspective in facilitating the regulatory path for cancer immunotherapy products and strengthening the regulatory framework around these very interesting and exciting technologies.

However, these criticisms do not substantially detract from a book that will prove extremely useful as an introduction to the rapidly evolving field of cancer immunotherapy with a focus on overcoming tumor-derived immunosuppression and evasion. 